\title{
Steady moving contact line of water over a no-slip substrate
}

\section{Challenges in benchmarking phase-field and volume-of-fluid methods against molecular dynamics simulations}

\author{
Uğis Lācis ${ }^{1, a}$, Petter Johansson ${ }^{2}$, Tomas Fullana ${ }^{3}$, Berk Hess ${ }^{2}$, Gustav Amberg ${ }^{1,4}$, \\ Shervin Bagheri ${ }^{1}$, and Stephané Zaleski ${ }^{1,3}$ \\ ${ }^{1}$ FLOW Centre, Department of Engineering Mechanics KTH, 10044 Stockholm, \\ Sweden \\ 2 Swedish e-Science Research Centre, Science for Life Laboratory, Department of \\ Applied Physics KTH, 10044 Stockholm, Sweden \\ ${ }^{3}$ Sorbonne Université and CNRS, Paris, France \\ ${ }^{4}$ Södertorn University, Stockholm, Sweden
}

Received 19 December 2019 / Accepted 6 July 2020

Published online 14 September 2020

\begin{abstract}
The movement of the triple contact line plays a crucial role in many applications such as ink-jet printing, liquid coating and drainage (imbibition) in porous media. To design accurate computational tools for these applications, predictive models of the moving contact line are needed. However, the basic mechanisms responsible for movement of the triple contact line are not well understood but still debated. We investigate the movement of the contact line between water, vapour and a silica-like solid surface under steady conditions in low capillary number regime. We use molecular dynamics (MD) with an atomistic water model to simulate a nanoscopic drop between two moving plates. We include hydrogen bonding between the water molecules and the solid substrate, which leads to a sub-molecular slip length. We benchmark two continuum methods, the Cahn-Hilliard phase-field (PF) model and a volume-of-fluid (VOF) model, against MD results. We show that both continuum models reproduce the statistical measures obtained from MD reasonably well, with a trade-off in accuracy. We demonstrate the importance of the phase-field mobility parameter and the local slip length in accurately modelling the moving contact line.
\end{abstract}

\section{Introduction}

The motion of a two-fluid interface contacting a flat solid surface poses a particularly difficult problem of continuum fluid mechanics. If the traditional point of view of a no-slip wall - a sharp transition between the phases and constant surface tension

\footnotetext{
a e-mail: ugis@mech.kth.se
} 
- is to be believed, then a contradiction ensues since at the triple point or contact line the velocity is both zero and non zero [1]. Attempts to solve this paradox and make progress on the issue abound [2-4]. One of the most popular is the assumption of Navier slip [5], but in general all solutions to the paradox amount to the introduction of a small length scale $l_{\mu}$ below which the continuum model ceases to be valid as discussed by Voinov [6]. Cox [7] extended Voinov's theory to arbitrary viscosity ratios and contact angles. Since then, many theoretical endeavours has been directed towards solving this problem [8-17] and the effort continues. Nevertheless, the microscopic scale physics remain somewhat mysterious as described for example in the review by Blake [18]. Indeed, and beyond the paradox described above, there are many uncertain features of the nanoscopic flow and interface shape: there is uncertainty about the value of the contact angle at the smallest scale, and about the nature of the deviation from equilibrium, the effect of molecular forces, the presence of evaporation, etc. Despite recent advancements [19], experiments have difficulty providing interface shape and velocity field data for moving contact lines below the micron scale. From the applied mathematical point of view, Navier slip regularisation leads to an approximation in which the curvature still diverges logarithmically at the contact line and to a contradiction if the velocity field is continuous [20,21]. Moreover certain fluid and surface combinations have very small slip lengths, below the nanometer scale $[22,23]$. In such systems, if one considers the problem at smaller and smaller scales, other molecular effects will become relevant before the slippage effects.

Thus a full experimental characterisation at the nanometer scale is difficult and it very well may be that, at least for some time, only molecular dynamics (MD) "numerical experiments" will provide the insight necessary to understand which regularisation is adequate, and particularly so when the slip length is small and other effects than slip are required for a well-posed problem. However the largest possible MD simulations in three dimensions are limited in physical size to only tens of nanometers in each direction. This would make it impossible to perform asymptotic matching with numerical solutions of the Navier-Stokes equations. Indeed, in order to perform the matching over all scales necessary, the Navier-Stokes equations would have to be solved down to the nanometer scale. Moreover, for a $1 \mathrm{~mm}$ droplet, refining the calculation to the nanometer scale would require a range of scales of $10^{6}$, which implies the impossible-to-attain number of $10^{18}$ grid points. Even restricting the computations to two dimensions of space, $10^{12}$ grid points sit on the borderline of currently feasible computations (and not all problems warrant the use of a supercomputer). This creates the necessity either for investigations limited to much smaller scales, technically adapted approaches to to enable the hybrid method for matching MD and Navier-Stokes solvers [24-27], or for an intermediate-scale model, between the molecular scales and the scale of the sharp-interface model. Such an intermediate scale model may be provided by the diffuse interface approach, in which the hypothesis of a sharp transition between phases is replaced by that of a smooth transition over a small length scale $\epsilon$. In the words of Pismen [28] "Multiscale methods employing different techniques at disparate length and time scales is the only feasible way to overcome the scale gap in practical computations". Simultaneous usage of MD, a diffuse interface model and a sharp interface model would be an implementation of this program, with the diffuse interface approach playing a key role at the intermediate scale. In such a framework, MD would help design and define parameters for the diffuse interface model, which in turn would perform a similar service for larger scale sharp interface models.

Beyond offering an intermediate scale, diffuse interface models open the possibility of regularising the contact line problem in an efficient and physically meaningful way. Indeed, together with MD, they have led to a generalisation of the Navier slip boundary condition (so called GNBC) that takes into account the "uncompensated 


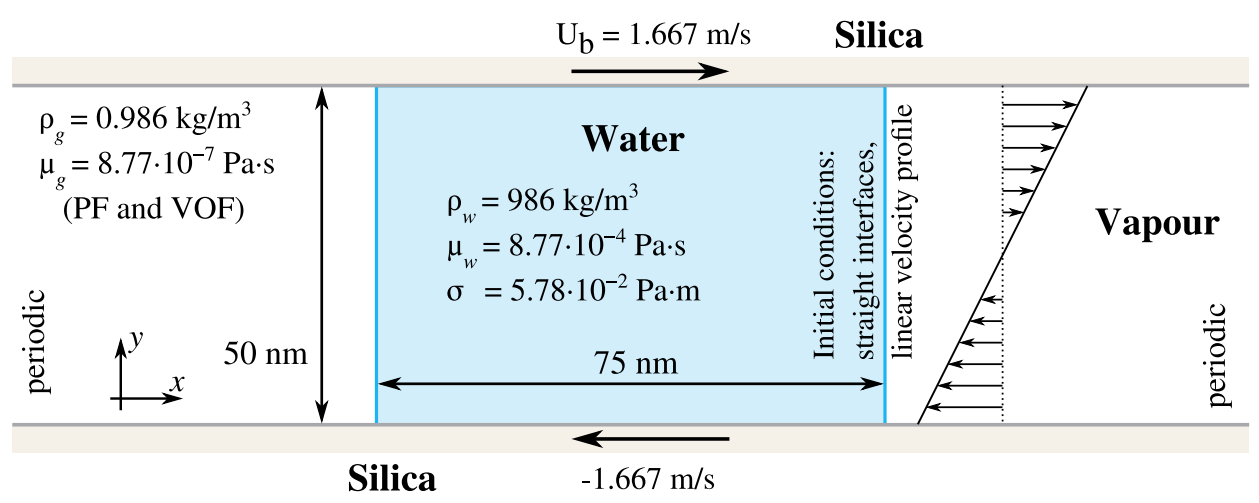

Fig. 1. Illustration of the two-phase forced wetting configuration. Water parameters are determined from the molecular dynamics water model, at temperature $T=300 \mathrm{~K}$. The vapour parameters for phase field and volume-of-fluid models are 1000 times smaller. The chosen parameters lead to capillary number $\mathrm{Ca}=2 \mu_{w} U_{b} / \sigma=0.05$.

Young's stress" ${ }^{1}$ and makes the contact line motion problem well posed [30,31]. On the other hand, diffuse interface models $[32,33]$ introduce at least two additional length scales, the interface thickness $\epsilon=\beta / \alpha$ and the diffusion length $l_{d}=\sqrt{\mu_{l} M}$ (the reader is referred to Section 3 and Appendix B for a presentation of the diffusionbased phase-field (PF) model parameters). The number of length scales is different, for example, for van der Waals model [34] or Cahn-Allen model [35]. Since the newly introduced length scales come in addition of the slip length $l_{s}$ there are potentially three length scales that can play an important role in the physics of the contact line. In addition a diffuse interface model has several other parameters specifically related to the contact line: the so called contact line friction $[32,36]$, the surface energies (that lead to the equilibrium contact angle through Young's relation) and their spatial distribution. This makes the selection of the parameters rather difficult: there are three length scales and two or more other parameters to adjust or select. In a way, this is the price to pay to have a more realistic continuum description of the nanoscale.

This motivates our choice of a physical situation where two simplifications occur: small slip and negligible evaporation. Indeed, for water on silica far from the critical point, slip is relatively small and evaporation is moderate. This situation may be reproduced using $\mathrm{MD}$ based on the $\mathrm{SPC} / \mathrm{E}$ water model, which allows us to capture the strong hydrogen bonds between the water molecules and silica molecules on the surface [22]. Many possible application targeted configurations can be investigated, such as capillary driven flows [37] or forced wetting flows [18]. For the purpose of this work, we use a two-dimensional water drop enclosed by two moving walls, as shown in Figure 1 at sufficiently small capillary number $\mathrm{Ca}=2 \mu_{w} U_{b} / \sigma=0.05$ for existence of steady state configuration $[38,39]$. Here, $U_{b}$ is wall velocity, $\mu_{w}$ is water viscosity and $\sigma$ is surface tension. The choice of water in contact with a silica-line substrate makes the work reported here markedly different from previous MD investigations of the contact line dynamics in the same geometrical configuration $[18,30,40]$. The chosen geometrical configuration is also known for its simplicity: it involves a symmetrical setting with identical solid walls providing us with identical two phase interfaces. Using this configuration we report on a first attempt to match MD, PF, and volume-of-fluid (VOF) simulations. By matching we mean finding the PF or VOF

\footnotetext{
${ }^{1}$ The inception of the GNBC is sometimes attributed to Blake [29], who discuss the uncompensated Young's stress and slippage velocity in context of adsorption and desorption modelling.
} 
parameters that best reproduces the steady shape of the interface and the steady velocity field from the MD. This procedure is, as far as we know, original. Several other studies of the correspondence between the PF parameters [41] or the VOF parameters [42] and MD were performed, but not the direct attack on the contact line dynamics problem on a no-slip substrate as we suggest here.

This paper is organised as follows. In Section 2, we describe the MD simulations and the measurements, later used for the benchmarking of PF and VOF. Then, in Section 3, we describe the PF model we use, the matching procedure to reproduce the MD results and show how does $\mathrm{PF}$ and MD compare. The VOF model and comparison between the VOF and MD is shown in Section 4. Next, in Section 5, we discuss the presented results and some open questions that remain. Finally, in Section 6, we draw conclusions from this study.

\section{Molecular dynamics simulations of water over silica-like substrate}

Molecular dynamics describes the system on a molecular level. The simulated system consists of water molecules, which interact with other water molecules as in the system as well as the substrate through a specified force field. Molecule positions and velocities are then integrated over time and the results sampled to obtain a representation of continuum variables.

\subsection{Setup}

To represent the chosen water-vapour geometry (Fig. 1), a two-dimensional shear system with water in vacuum is created by placing a water slab between two walls, as shown in Figure 2a. After the initial equilibration step, some of the water molecules move to the void and essentially form a very sparse water vapour. The walls consist of rigid $\mathrm{SiO}_{2}$ molecules which are neutral electric quadrupoles. Water cannot easily slip over this substrate due to the electrostatic interactions between water molecules, which are dipoles, and the substrate quadrupoles. Water forms hydrogen bonds with this substrate, a form of bond that is transient but strong enough to prohibit slip [23]. Due to the local nature of the hydrogen bonding, only one layer of the solid substrate is sufficient to capture the physics of the moving contact line. The SPC/E model is the simplest possible choice of a MD model capable of describing the hydrogen bonding. A more complex water model would improve the agreement between the physical properties of MD and real water, but would not yield fundamentally different results for contact line motion. The surface-water interaction is tuned to yield equilibrium contact angle $\theta_{0}=97.5^{\circ}$.

Data is collected inside bins of size $0.25 \times 0.25 \mathrm{~nm}^{2}$ along $x$ and $y$. These bins form a regular grid covering the entire system. The binning is visualised along the $y$ axis in Figure 2b. Average water molecule velocity, mass and temperature is sampled inside all bins over a period of $50 \mathrm{ps}$, after which the data is stored and the bins are reset for the next sampling period.

We perform four simulations from different starting configurations. These configurations are created by generating the initial velocity field with different seeds for the random generator. Before the shear is applied each configuration is allowed to relax over a period of 100 ps. Evaporation and condensation process is a liquid surface effect and we have observed that $100 \mathrm{ps}$ is sufficient to reach equilibrium vapour density. More details about the procedure are available in Appendix A. 
(a)

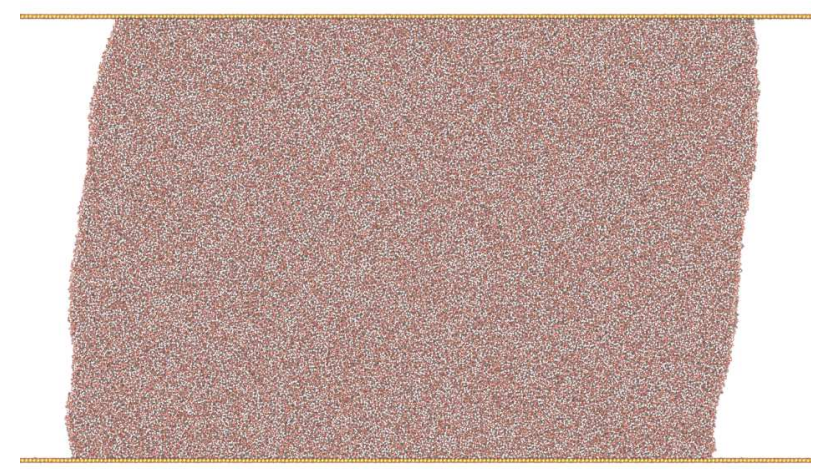

(b)
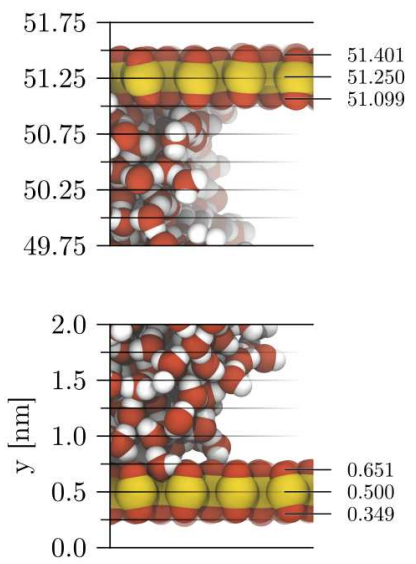

Fig. 2. (a) Molecular view of the sheared water droplet configuration in steady regime. (b) Close up on upper and lower contact lines. The binning for measurements from MD along the $y$ axis is $0.25 \mathrm{~nm}$ and depicted with horizontal lines. The water slab begins in the fourth bins from the bottom and top. The coordinates of the wall atoms are marked on the right.

\subsection{System parameters}

Parameters for the MD system are reported in Figure 1. Bulk water density $\rho_{w}$, viscosity $\mu_{w}$ and surface tension $\sigma$ are measured in separate simulations of pure water. Following Quan et al. [30] the water slip over the substrate is characterised through a friction parameter $\beta_{f}=\mu_{w} / l_{s}$, where $l_{s}$ is the corresponding Navier slip length. The friction parameter $\beta_{f}=5.9 \mu_{w} \mathrm{~nm}^{-1}$ is measured in a Couette flow setup using the boundary layer of water molecules in direct contact with the wall molecules. From this measurement, the Navier slip length $l_{s}=0.17 \mathrm{~nm}$ is extracted. Finally, the interface width $\epsilon$ is taken as the length over which the density goes from bulk to vapour density. This change occurs over three bins, giving $\epsilon=0.75 \mathrm{~nm}$.

\subsection{Results}

Shear experiments consist of two stages. Once the shear is applied the water slab deforms until reaching its equilibrium (steady state). For the transient state we measure the separation $\Delta x(t)$ between the upper and lower contact line positions, which begins at zero in the original slab geometry and increases to $\Delta x_{s}(t)$ in the steady state. To identify the interface between the water phase and vapour phase, we locate the bins with a water density measurement, which is half of the bulk density. The mean (averaged between four different runs) separation for the four simulations is shown in Figure 3a with solid line. The steady state is reached in 5.9 ns, after which the data has been averaged in time to obtain the final mean displacement $\Delta x_{m}=5.89 \mathrm{~nm}$.

In the steady state the slabs are characterised using the full interface shape $x(y)$ and the flow field. We mirror the right interface along both axes and overlay it on top of the left interface. Thus the lower end represents the receding contact line and the upper end the advancing. The average of all interfaces from MD in the steady regime is shown in Figure 3b. To gain more detailed insight into the agreement between $\mathrm{MD}$ and continuum models, we introduce parametric coordinate $s$ as illustrated in 
(a)

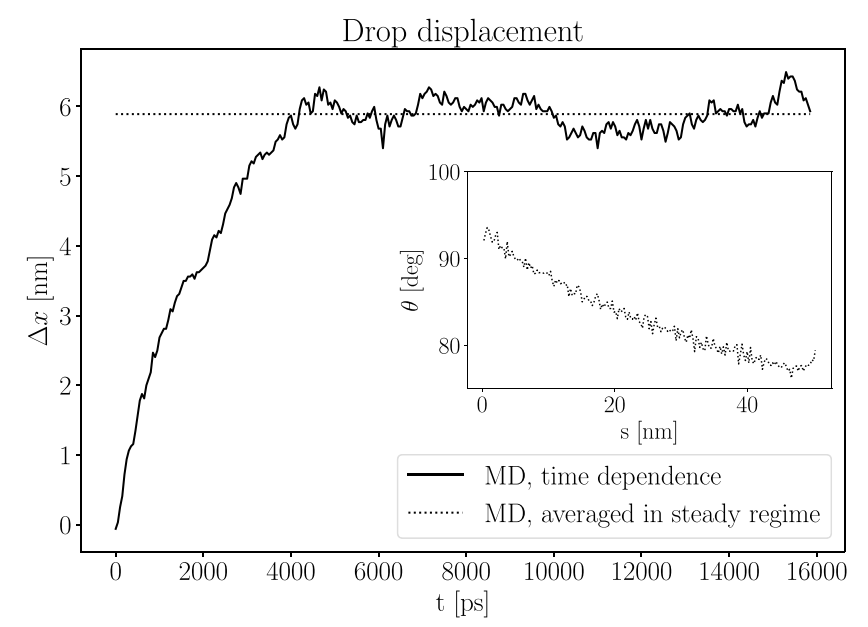

(b)

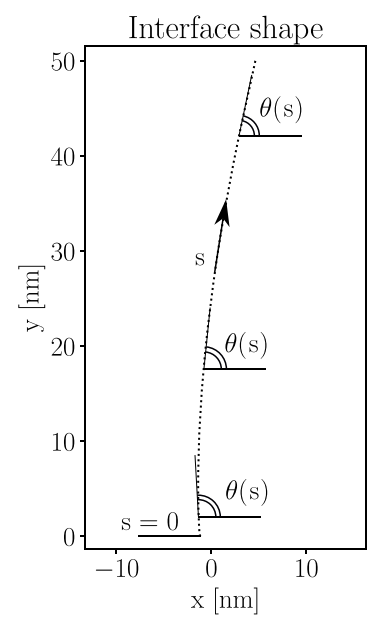

Fig. 3. (a) Shear separation $\Delta x(t)$ from the four MD simulations with mean value $\Delta x=$ $5.89 \mathrm{~nm}$ in the steady regime. (b) Average interface shape in the steady state. The average is done around the midpoint, at $y=25 \mathrm{~nm}$. As additional measure, we extract the interface angle along the curvilinear coordinate $s$ as illustrated in (b). The interface angle from MD is shown in the inset of (a).

Figure 3b. The interface angle $\theta(s)$ is shown in the inset of Figure 3a. Finally, we extract an averaged flow field from the MD runs, which we will use to discuss the agreement between MD, PF and VOF (Figs. 4, 5, 6, 7 and 10).

\section{Phase-field model}

We consider a 2D phase-field (PF) model of a binary mixture to model two regions of different densities and viscosities. The water and vapour phases in Figure 1 are assumed to be incompressible and the interface between the regions to be diffuse, i.e. that quantities vary smoothly over the interface.

\subsection{Governing equations for the binary mixture}

The phase-field model introduces a phase variable $C(x, y)$ ranging from 1 in the water phase to -1 in the vapour. The derivation of the governing equations for the phase variable can be found in $[33,43]$. The phase-field variable is governed by a convection-diffusion equation in a yet undetermined flow field $\mathbf{u}$ as

$$
\frac{\partial C}{\partial t}=\nabla \cdot\left[M \nabla\left(\beta \Psi^{\prime}(C)-\alpha \nabla^{2} C\right)\right]-\mathbf{u} \cdot \nabla C .
$$

The diffusive flux $\mathbf{J}_{d}=-M \nabla \phi$ is proportional to the gradient of the chemical potential, defined as $\phi=\beta \Psi^{\prime}(C)-\alpha \nabla^{2} C$, with proportionality coefficient $M$, which is called the phase-field mobility. Due to the assumption of an incompressible flow, the convective flux takes the simple form $\mathbf{J}_{c}=\mathbf{u} \cdot \nabla C$. In the chemical potential, we have two parameters $\alpha$ and $\beta$, which are related to the surface tension as $\sigma=2 \sqrt{2 \alpha \beta} / 3$ and the characteristic thickness of the diffuse interface as 
$\epsilon=\sqrt{\alpha / \beta}$. In addition, it contains the derivative of the standard double-well potential $\Psi(C)=(C+1)^{2}(C-1)^{2} / 4$.

The motion of the fluids is described by the incompressible Navier-Stokes equations with variable density and viscosity. We define the density and viscosity as

$$
\rho(C)=\rho_{w} \frac{C+1}{2}-\rho_{g} \frac{C-1}{2} \quad \text { and } \quad \mu(C)=\mu_{w} \frac{C+1}{2}-\mu_{g} \frac{C-1}{2},
$$

respectively. Here $\rho_{w}$ and $\mu_{w}$ are the density and the viscosity of the water, and $\rho_{g}$ and $\mu_{g}$ are the density and the viscosity of the vapour component. The Navier-Stokes equations then become

$$
\begin{aligned}
\rho(C)\left[\frac{\partial \mathbf{u}}{\partial t}+(\mathbf{u} \cdot \nabla) \mathbf{u}\right] & =-\nabla P+\nabla \cdot\left[\mu(C)\left\{\nabla \mathbf{u}+(\nabla \mathbf{u})^{T}\right\}\right]+\mathbf{f}_{\sigma}, \\
\nabla \cdot \mathbf{u} & =0
\end{aligned}
$$

where the volume force $\mathbf{f}_{\sigma}=-C \nabla \phi$ corresponds to the surface tension force and acts over the diffuse interface region. This form of the surface tension forcing is the so called potential form [44], which uses a reduced pressure.

\subsection{Boundary conditions for the phase-field model}

The convection-diffusion equation (1) is a fourth-order partial differential equation and requires two boundary conditions. First, we impose a non-equilibrium wetting condition $[30,32,33]$ on the solid moving wall,

$$
-\mu_{f} \epsilon\left(\frac{\partial C}{\partial t}+\mathbf{u} \cdot \nabla C\right)=\alpha \nabla C \cdot \hat{n}-\sigma \cos \left(\theta_{0}\right) g^{\prime}(C),
$$

where $\mu_{f}$ is a contact line friction parameter, having the same units as bulk dynamic viscosity. Here, $\theta_{0}$ is the equilibrium contact angle and $g(C)=0.5-0.75 C+0.25 C^{3}$ is a switching function describing a smooth transition from water to vapour. The unit normal vector $\hat{n}$ is directed from the fluid to the surrounding solid, which is standard definition in numerical approaches. If one sets $\mu_{f}=0$, the dynamic contact angle is always enforced to the equilibrium angle $\theta_{0}$. Non-zero contact line friction allows the dynamic contact angle to evolve naturally as a function of contact line speed. The second boundary condition for the phase function is zero diffusive flux of chemical potential through the boundaries, i.e. $\nabla \phi \cdot \hat{n}=0$. On the outer sides of the domain, periodic boundary conditions are enforced.

The fluid momentum equations are subject to zero wall-normal velocity, $u_{y}=0$. For the tangential velocity component, we first consider the classical no-slip condition, which is equivalent to setting the fluid velocity near the moving wall to the velocity of the wall, $u_{x}=U_{w}$. In this situation, the only mechanism through which the contact line can move is the diffusion of the phase-field variable. We also impose the Navier slip condition, $u_{x}=U_{w}-l_{s} \partial_{y} u_{x} \hat{n}_{y}$, where $l_{s}$ is the Navier slip length. The final boundary condition which we investigate for the PF model is the generalised Navier boundary condition (GNBC). This boundary condition (using the friction factor $\beta_{f}$ for the slip velocity [30]) takes the form

$$
\beta_{f}\left(u_{x}-U_{w}\right)=-\mu \partial_{y} u_{x} \hat{n}_{y}+\left[\alpha \partial_{y} C \hat{n}_{y}-\sigma \cos \left(\theta_{0}\right) g^{\prime}(C)\right] \partial_{x} C
$$


where the second term is the uncompensated Young's stress. We assume a constant slip length $l_{s}$ over the whole solid surface. This leads to a friction coefficient $\beta_{f}$, which varies in the same way as the liquid viscosity. The equations are implemented in a finite-element solver (for more details see Appendix B).

\subsection{Comparison with molecular dynamics}

The PF parameters contain quantities uniquely determined from $\mathrm{MD}\left(\rho_{w}, \mu_{w}, \sigma\right.$, $\left.\theta_{0}, \epsilon\right)$ and quantities that are less known or fully unknown $\left(\mu_{f}, M, \rho_{g}\right.$ and $\left.\mu_{g}\right)$. The vapour properties are not known due to the small system size, which renders any measurements from the MD outside of the liquid phase impractical. Therefore we have fixed both $\rho_{g}$ and $\mu_{g}$ in the PF (and also later in the VOF) simulations as small as numerically feasible, $\rho_{g}=10^{-3} \rho_{w}$ and $\mu_{g}=10^{-3} \mu_{w}$, see also Figure 1. The limiting factor for our implementation is viscosity ratio. The small density and viscosity in vapour phase is motivated by the fact that in MD there are only a handful of vapour molecules outside the water droplet (Appendix A), and those consequently does not exhibit any notable stress on the liquid phase. An alternative approach could be to read off the density and viscosity data from measurements $[45,46]$, which would yield $\rho_{g}=2.64 \cdot 10^{-5} \rho_{w}$ and $\mu_{g}=1.13 \cdot 10^{-2} \mu_{w}$. We have checked that using density and viscosity from literature for PF simulations yields only minor changes and does not affect the conclusions of this study. The remaining unknown parameters $\left(\mu_{f}\right.$ and $M)$ are fitted to the MD data.

The fitting procedure I, which we later refer to as "fit I", is as follows:

1. adjust the contact line friction $\left(\mu_{f}\right)$ individually for advancing and receding contact lines to match the MD dynamic contact angles;

2. adjust the phase-field mobility $(M)$ to match the MD drop displacement $\Delta x_{m}$.

Results are shown in Figures 4a and 4b. There is a local error in the interface angle (Fig. 4b) near the advancing contact line. The drop displacement from PF agrees with $\Delta x_{m}$ with an accuracy of $2 \%$, which we consider a very good match. The parameters needed to arrive with the corresponding PF results along with the obtained drop displacement are listed in Table 1.

A $10 \mathrm{~nm} \times 10 \mathrm{~nm}$ close-ups of MD streamlines near the advancing and the receding contact lines are shown in Figures $5 \mathrm{a}$ and 5c, respectively. For comparison, in Figures $5 \mathrm{~b}$ and $5 \mathrm{~d}$ we show $20 \mathrm{~nm} \times 10 \mathrm{~nm}$ close-ups of streamlines from $\mathrm{PF}$ with GNBC. We observe that PF streamlines cross the interface and extend several nanometers into the vapour. This is in contrast to the MD results and therefore the PF predictions obtained through fitting procedure I provide un-physical flow fields. The PF flow with no-slip and Navier-slip conditions show slightly worse agreement and are not reported. The extent of which PF streamlines cross from water to vapour in the $\mathrm{PF}$ results can be characterised with the Pe number (Appendix B), quantifying the relative importance between convection and diffusion and defined as $\mathrm{Pe}=U \epsilon L /(M \sigma)$. From the last row in Table 1 we observe that all fits have resulted in a $\mathrm{Pe}$ which is much smaller than unity: a high diffusion regime, in contrast to the convection dominated MD data. The high diffusion leads to the many streamlines crossing the PF interface. However, if one would use a much larger Pe number (much smaller mobility) but keep other parameters the same, the MD steady state drop displacement $\Delta x_{m}$ would be largely overestimated.

To obtain a more physical flow field, we devise another fitting procedure, in which we relax the requirement on the dynamic contact angle. Fitting procedure II ("fit II") is defined as: 
(a)

Interface shape, fit I

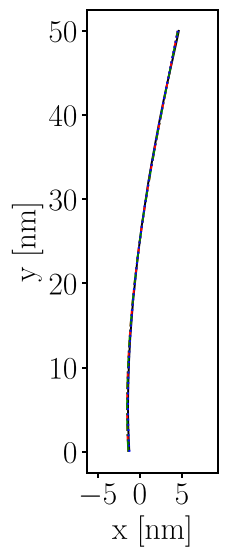

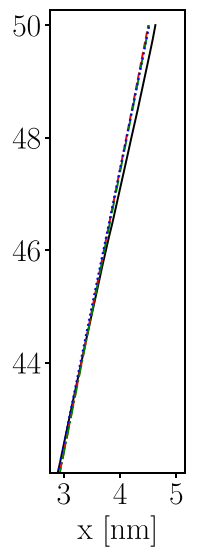

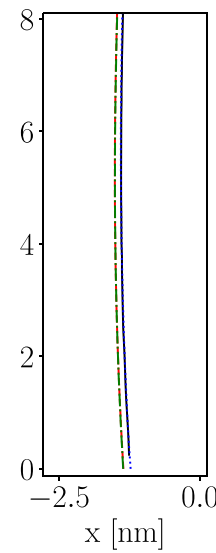

(b)

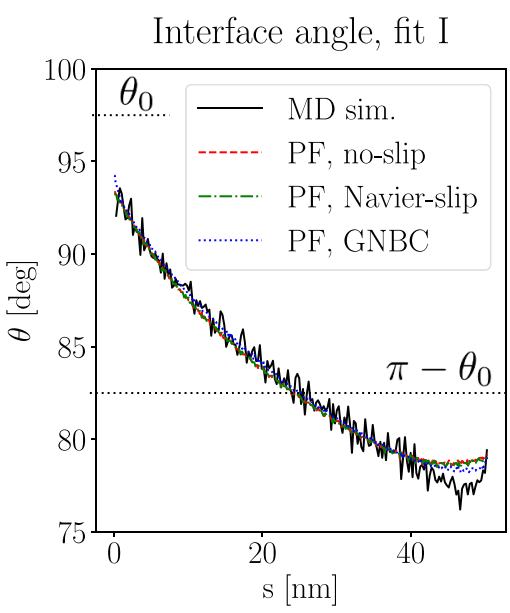

Fig. 4. Results of the fitting procedure I (accurate dynamic contact angle). Steady interface shape (a) and angle distribution over the interface (b) from MD simulations and fitted PF simulations with different boundary conditions. Static contact angles for advancing and receding contact lines are indicated with dotted black lines.

Table 1. Summary of the obtained phase-field parameters and displacement, fit I.

\begin{tabular}{lllll}
\hline & PF no-slip & PF Navier-slip & PF GNBC & MD \\
\hline$\mu_{f a} / \mu_{w}$ & 2.5 & 2.8 & 12 & - \\
$\mu_{f r} / \mu_{w}$ & 3.0 & 3.3 & 13 & - \\
$\mathrm{Pe}=U \epsilon L /(M \sigma)$ & 0.070 & 0.070 & 0.075 & - \\
$\Delta x$ & $5.85 \mathrm{~nm}$ & $5.87 \mathrm{~nm}$ & $5.75 \mathrm{~nm}$ & $5.89 \mathrm{~nm}$ \\
\hline
\end{tabular}

(a) MD adv. (b) PF GNBC adv.

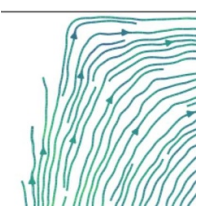

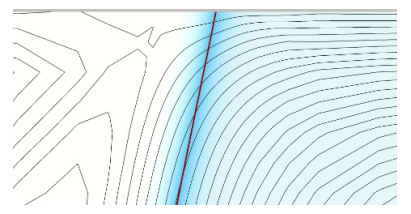

(c) MD rec.

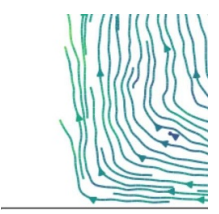

(d) PF GNBC rec.

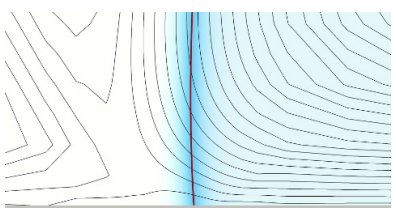

Fig. 5. Flow field results of the fitting procedure I. Streamlines near advancing contact line $(a, b)$ and near receding contact line (c,d) from MD simulations (a,c) over a $10 \mathrm{~nm} \times 10 \mathrm{~nm}$ patch and PF simulations with GNBC boundary condition (b,d) over $20 \mathrm{~nm} \times 10 \mathrm{~nm}$ patch. With red line, we indicate the isoline of $C=0$, which represents the interface. The light blue background field show the variation of the phase-field variable $C$.

1. for PF simulation with no-slip boundary conditions, we select $\mu_{f}=\mu_{w}$ and then vary $M$ to match the drop displacement $\Delta x_{m}$ observed in MD;

2. for PF simulations with Navier-slip and GNBC boundary conditions, we adjust $\mu_{f}$ to match the contact angle in the final no-slip simulation, carried out in point 1. of this strategy, and then fit $M$ to match the drop displacement $\Delta x_{m}$ from the MD. 
(a)

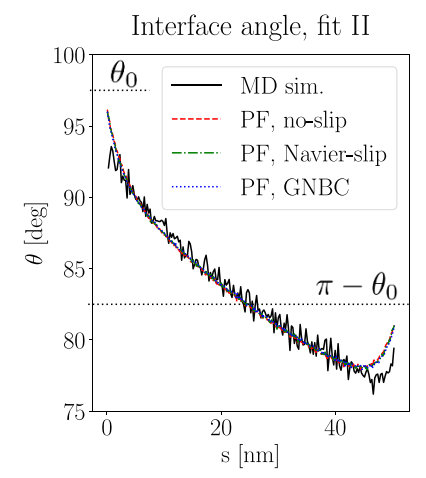

(b) MD

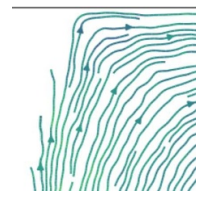

(d) PF Navier-slip

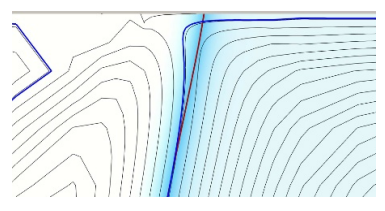

(c) PF No-slip

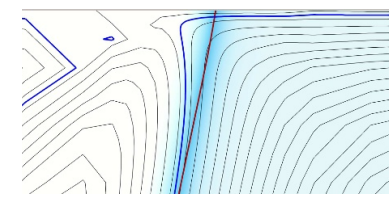

(e) PF GNBC

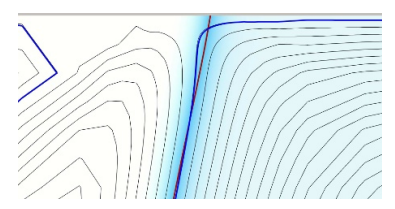

Fig. 6. Results of the fitting procedure II (larger error on dynamic contact angle). Angle distribution over the interface (a) from MD simulations and fitted PF simulations with different boundary conditions. Streamlines near advancing contact line (b-e) from MD simulations (b) over a $10 \mathrm{~nm} \times 10 \mathrm{~nm}$ patch and PF simulations with different boundary conditions (c-e) over $20 \mathrm{~nm} \times 10 \mathrm{~nm}$ patch. With red line, we indicate the isoline of $C=0$, which represents the interface. The dark blue line represents a streamline originating $0.25 \mathrm{~nm}$ away from the wall within the liquid drop.

Table 2. Summary of the obtained phase-field parameters and displacement, fit II.

\begin{tabular}{lllll}
\hline & PF no-slip & PF Navier-slip & PF GNBC & MD \\
\hline$\mu_{f a} / \mu_{w}$ & 1.0 & 1.1 & 2.0 & - \\
$\mu_{f r} / \mu_{w}$ & 1.0 & 1.1 & 2.0 & - \\
$\mathrm{Pe}=U \epsilon L /(M \sigma)$ & 0.8 & 1.4 & 1.8 & - \\
$\Delta x$ & $5.84 \mathrm{~nm}$ & $5.82 \mathrm{~nm}$ & $5.81 \mathrm{~nm}$ & $5.89 \mathrm{~nm}$ \\
\hline
\end{tabular}

We carry out these fits for all considered boundary conditions. By investigating the obtained interface angle distribution (Fig. 6a) we see that overall agreement is good, while the local error near contact lines is increased. This difference is, however, not visible in the interface shape: practically the same agreement as presented in Figure 4a is obtained. However, investigating the flow field near the advancing contact line reveals much better agreement with the MD results, although there is a small overshoot through the interface of streamlines close to the wall (for fair measurement of the overshoot, we have identified a single streamline in all the simulations, which originates $0.25 \mathrm{~nm}$ away from the wall within the drop). The improved agreement with the MD (and smaller overshoot of the streamlines) is due to a smaller phasefield mobility (or larger Pe number, see Tab. 2). The reduced contact line friction is the reason why it is possible to use smaller phase-field mobility. Reducing contact line friction leads to smaller displacement of the drop, which consequently allows to reduce the phase-field mobility to increase the drop displacement back to the $\Delta x_{m}$.

These results suggest that as the phase-field mobility $M$ is reduced (or Pe number is increased), the flow field near contact line approaches the one observed in MD. Therefore we devise the final fitting procedure III ("fit III"), which is defined as:

1. set the contact line friction $\mu_{f}=0$ (fixing the dynamic contact angle to the equilibrium one). This gives minimum friction at the contact line;

2. fit the phase-field mobility $M$ to obtain the drop displacement $\Delta x_{m}$. 
Table 3. Summary of the obtained phase-field parameters and displacement, fit III.

\begin{tabular}{llll}
\hline & PF no-slip & PF Navier-slip & MD \\
\hline$\mu_{f a} / \mu_{w}$ & 0.0 & 0.0 & - \\
$\mu_{f r} / \mu_{w}$ & 0.0 & 0.0 & - \\
$\mathrm{Pe}=U \epsilon L /(M \sigma)$ & 2.9 & 6.0 & - \\
$\Delta x$ & $5.84 \mathrm{~nm}$ & $5.82 \mathrm{~nm}$ & $5.89 \mathrm{~nm}$ \\
\hline
\end{tabular}

(a)

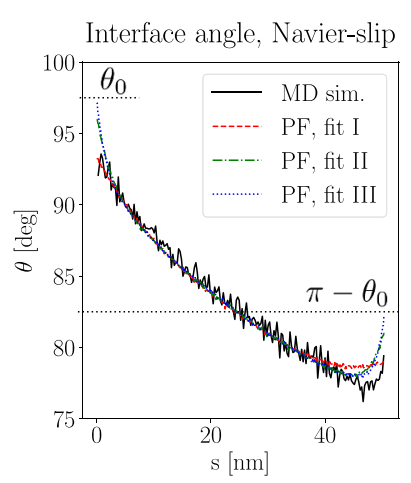

(b) MD

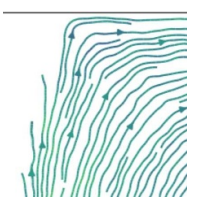

(d) PF Navier-slip fit II

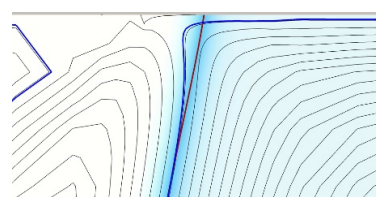

(c) PF Navier-slip fit I

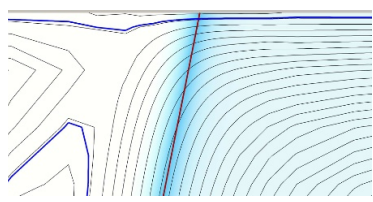

(e) PF Navier-slip fit III

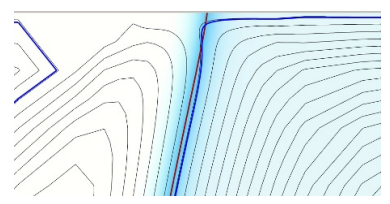

Fig. 7. Compilation of PF simulation results with Navier-slip boundary condition, including results from fit III. Same measures are reported as in Figure 6.

The obtained Pe numbers using fitting procedure III are given in Table 3. The results from PF simulations with Navier-slip boundary condition from all fitting procedures are shown in Figure 7. For the fitting procedure III it is meaningless to use GNBC, since the GNBC is equivalent to Navier-slip boundary condition for $\mu_{f}=0$. In Figure 7a we see the increasing local error of angle near the contact lines, which remains localised in a thin region near both walls. By investigating the streamlines (Figs. 7b-7e) we conclude that indeed as the PF mobility is reduced, the overshoot of the streamlines in PF is reduced and consequently the agreement with MD improves. Similar conclusion can be drawn from no-slip PF simulations.

\section{Volume-of-fluid model}

The VOF model is known to be well-suited for solving interfacial flows. The interface between water and vapour in VOF - in contrast to the PF - is reconstructed in a sharp manner. Therefore the VOF model is a good candidate and is typically used to solve two-phase flows in macroscopic systems. In this section, we investigate how accurately the model can capture the behaviour of the nanoscopic droplet.

\subsection{Governing equations for the two-phase flow with VOF model}

In the VOF method, the fluid momentum is governed by exactly the same equations as in the PF method, namely, incompressible Navier-Stokes equations with variable density and viscosity (3)-(4). The difference in methods lies in three aspects. First, the governing equation for the concentration function $C$, which within VOF method 
is typically called volume fraction, is a convection equation

$$
\frac{\partial C}{\partial t}+\mathbf{u} \cdot \nabla C=0
$$

instead of the convection-diffusion equation (1) for the PF concentration. Second, the volume fraction $C$ varies from 0 in the vapour phase to 1 in the fluid phase and consequently fluid density and viscosity becomes

$$
\rho(C)=C \rho_{w}+(C-1) \rho_{g} \quad \text { and } \quad \mu(C)=C \mu_{w}+(C-1) \mu_{g}
$$

respectively. The third difference lies in the fact that the surface tension force within the momentum equation (3) takes form

$$
\mathbf{f}_{\sigma}=\sigma \kappa \delta_{s} \hat{n}
$$

where $\kappa$ is the local curvature of the interface, $\delta_{s}$ is a discrete Dirac distribution function used to spread the surface tension force to the fluid mesh and $\hat{n}$ is the normal of the two-phase interface. The curvature and interface normal are approximated using the Continuum-Surface-Force approach, which states that

$$
\kappa \approx \nabla \cdot \hat{n} \quad \text { and } \quad \hat{n} \equiv \frac{\nabla C}{|\nabla C|} .
$$

The accuracy of the surface tension term is directly dependent on the accuracy of the curvature calculation. The height-function methodology is a VOF-based technique for calculating interface normals and curvatures. The interested reader can find more details on the VOF method in Appendix C.

\subsection{Boundary conditions for the VOF model}

For the VOF model used in this work, we use a similar set of boundary conditions as for the PF model. As we have two sets of unknowns (flow field and volume fraction), we need boundary conditions to determine both. Due to the fact that we are interested in steady regime only, we use a constant angle wetting condition. The angle is imposed through modification of height functions near the boundary and essentially sets the orientation of interface normal as illustrated in Figure 8. The imposed contact angle affects the overall flow calculation in two ways. First, it defines the orientation of the VOF interface reconstruction in cells that contain the contact line and, second, it influences the calculation of the surface tension term by affecting the curvature computed in cells at and near the contact line.

The second boundary condition is the velocity condition for the fluid flow. Although the VOF method does not allow for any diffusive transport of the contact line, there is always some mesh dependent numerical slip of the contact line [47]. This, however, does not provide accurate control over the results, therefore the explicit specification of a boundary condition compatible with a moving contact line is desired. As we have observed from PF result comparison with MD, the Navier-slip condition alone is sufficient to match the MD results, therefore we restrict the study on VOF only on the Navier-slip condition. Furthermore, since the observed slip length from the MD is very small - recall $l_{s}=0.17 \mathrm{~nm}$ - we made choice to localise the slip condition in the near vicinity of the moving contact line and away from the contact 


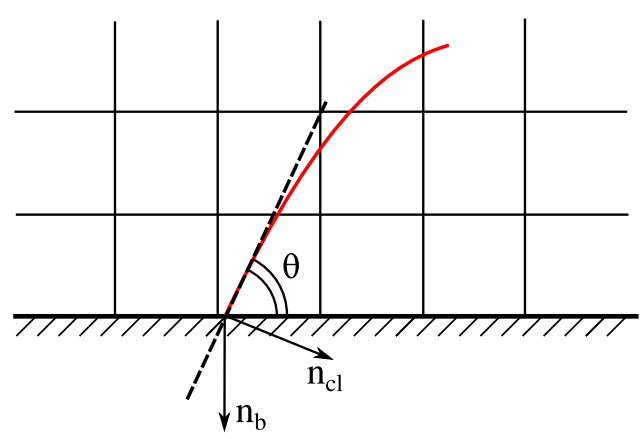

Fig. 8. Illustration of wetting condition employed for the VOF model. We use a constant contact angle setting that defines the normal $\hat{n}_{c l}$ of the interface at the contact line.

line impose the wall velocity $u_{x}=U_{w}$. The localised Navier-slip condition takes form

$$
u_{x}=U_{w}-f\left(\frac{d}{\epsilon_{b}}\right) l_{s} \partial_{y} u_{x} \hat{n}_{y}
$$

Here locality of the slip condition is enforced using the bell function

$$
f\left(\frac{d}{\epsilon_{b}}\right)=\left\{\begin{array}{cl}
{\left[\frac{1+\cos \left(\pi d / \epsilon_{b}\right)}{2}\right]^{2}} & |d|<\epsilon_{b} \\
0 & |d| \geq \epsilon_{b}
\end{array}\right.
$$

The bell function takes as an argument the distance from the contact line $d=x-x_{C L}$ and the width of the bell function $\epsilon_{b}$. For coordinates further than $\epsilon_{b}$ away from the contact line the function is set to zero to recover the no-slip condition. The wall normal velocity component is set to zero $u_{y}=0$, same as in PF. For more details on implementation of the velocity condition in the VOF model, see Appendix C.

\subsection{Comparison with molecular dynamics}

To obtain results from the VOF method, we carry out a fitting procedure, described as follows.

1. Fix the width of the bell function $\epsilon_{b}=3.91 \mathrm{~nm}$ or five grid sizes in order to capture the variation of the slip length with sufficiently many points.

2. Fix the advancing and receding contact angle to $\theta_{a}=101^{\circ}$ and $\theta_{r}=93^{\circ}$ to represent the dynamic angle in the steady regime.

3. Adjust the magnitude of the local slip length $l_{s}$ to match the drop displacement $\Delta x_{m}$ from the MD.

Note that for the wetting condition, ideally we would like impose some relationship or governing law relating contact angle and contact line velocity. However, because we are looking only at steady regime currently, we bypass the implementation of a proper dynamic contact angle model for simplicity.

Using this procedure, we have found that the local slip length, which provides the best match between the VOF and the MD is $l_{s}=8 \mathrm{~nm}$. In Figure 9 we show the interface shape and interface angle comparison between the VOF, PF and MD results. From Figure $9 \mathrm{a}$ we observe that interface shapes are practically indistinguishable. 
(a)

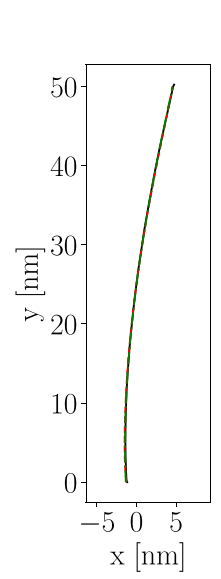

Interface shape

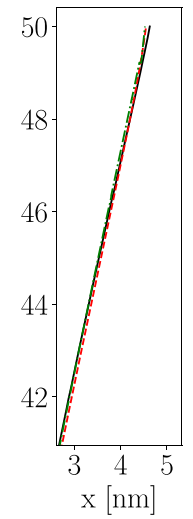

(b)

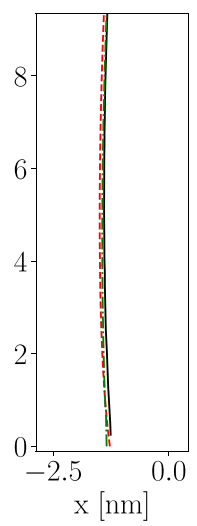

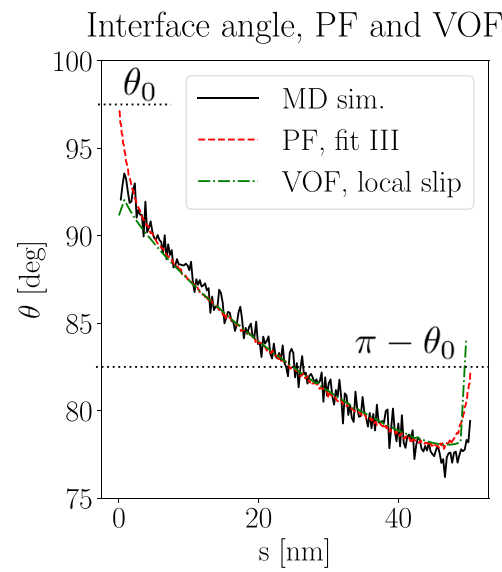

Fig. 9. Results of VOF simulations with local Navier-slip boundary condition in comparison with MD results and best PF results. Interface shape in (a) and angle distribution over the interface (b).

Table 4. Summary of the simulations carried out in this work, the corresponding boundary conditions in liquid and gas phases ("UYS" stands for uncompensated Young stress), as well as the mechanism of contact line motion (CLM) - either diffusion ("dif."), slippage ("slip") or a combination of both.

\begin{tabular}{lllll}
\hline & PF no-slip & PF Navier-slip & PF GNBC & VOF \\
\hline slip in vapour phase & $0.0 \mathrm{~nm}$ & $0.17 \mathrm{~nm}$ & $0.17 \mathrm{~nm}$ & $0.0 \mathrm{~nm}$ \\
slip in liquid phase & $0.0 \mathrm{~nm}$ & $0.17 \mathrm{~nm}$ & $0.17 \mathrm{~nm}$ & $0.0 \mathrm{~nm}$ \\
slip at contact line & $0.0 \mathrm{~nm}$ & $0.17 \mathrm{~nm}$ & $0.17 \mathrm{~nm}+$ UYS & $8.0 \mathrm{~nm}$ \\
CLM mechanism & dif. & dif., slip & dif., slip & slip \\
\hline
\end{tabular}

The interface angles from VOF, however, show similar local errors as the PF angles. Interestingly, for the first and last interface angle we observe a rather large jump in the VOF result, which might be an artefact of the constant contact angle imposition on the first mesh cell next to the wall.

\section{Discussion}

\subsection{The role of the velocity boundary condition}

As we have observed in the results of the PF simulations, the velocity boundary condition applied to the fluid momentum equation does not seem to be important for global measures, such as the drop displacement. Regardless of the imposed boundary condition, the drop displacement can be captured correctly. Therefore, for the VOF investigations, we have focused only on local Navier-slip boundary condition. For an overview of the fluid slippage conditions used in different types of simulations carried out in this work, we provide a summary in Table 4.

As for the interface shape, which is a more local measure, we have concluded that both benchmarked continuum models ( $\mathrm{PF}$ and VOF) had their shortcomings and the obtained interface angle always has some local errors near contact line. Notably, the $\mathrm{PF}$ has been successful in describing the interface angle at the receding contact 
line (Fig. 4b), but it always produced local error near the advancing contact line (for $s>40 \mathrm{~nm}$, Figs. 4b; 6a; 7a; 9b). The possible reasons for this disagreement could be an improperly chosen wall location as discussed in Section 5.2, and/or a misalignment of physics between the MD and PF as discussed in Section 5.3. Furthermore, when trying to match the interface angle as close to the MD as possible, we observe that the PF model provides non-physical flow field near the interface (Fig. 5), with streamlines crossing the interface and continuing in the vapour phase. This inaccuracy can be averted by allowing larger local errors for the interface angle (Figs. 6a and 7a), which then reduces the streamline crossing into the vapour phase significantly (Figs. 6c$6 \mathrm{e}$ and $7 \mathrm{c}-7 \mathrm{e})$. Note that the local error can be only observed when plotting the interface angle (Figs. 6a and 7a), while the interface shape is globally indistinguishable (Fig. 4a).

The improvement of the flow field was obtained irrespective of chosen boundary condition (Fig. 6c-6e), while the choice of the boundary condition introduce minor local improvements in either the flow field (if we demand the same accuracy of local interface angle near contact line) or in the local interface angle near contact line (if we demand the same accuracy of local flow field, set by the chosen PF mobility). The reason for improved flow field is that adding more slippage (first with Navier-slip, and then with GNBC) allows us to use smaller PF mobility $M$ and consequently obtain streamlines that follow the two phase interface more accurately.

For the best possible representation of the flow field, we have set the dynamic contact angle to the equilibrium values, which allowed us to use the smallest PF mobility parameter. This choice renders the GNBC condition obsolete. By comparing no-slip and Navier-slip conditions, Navier-slip has produced the most accurate streamlines with still acceptable representation of the interface shape. However, it is also possible to use the no slip condition with minor decrease of the flow field accuracy. Therefore our results indicate that the GNBC condition (or even Navier-slip condition) is not necessary for physically acceptable predictions for the flow system considered in the present work using the PF model. In addition, increase of the diffusive transport near the contact line seem to always worsen the flow field prediction, which suggests that sharp interface method could bet the best possible choice for modelling the present system.

For the accurate representation of the drop displacement in the VOF, we have fixed the width of the local Navier-slip condition and adjusted the amplitude. We have observed that using one particular slip length locally near the contact line allowed us to reach exactly the same drop displacement as MD. However, similar as for PF, the interface shape has small local errors in angle (Fig. 9), while interface shapes are globally indistinguishable.

\subsection{Wall location and slip condition}

To gain further insight into which boundary condition would be the most appropriate to model the system consisting of a water drop between two no-slip plates, we look at velocity distribution as a function of a distance from the contact line. The MD results are obtained as an average over the 4 runs centred to the moving contact line. The MD results are shown in Figure 10 with black crosses.

For comparison we show PF with Navier-slip condition, zero contact line friction $\mu_{f}=0$ and $\mathrm{Pe}=6$, which yielded the best fit of streamlines. Sampling the velocity field along the wall (Fig. 10, red dashed) provides unsatisfactory agreement with the MD both in the near vicinity of the contact line and also deeper in the drop. However, if the sampling location is moved $0.75 \mathrm{~nm}$ away from the wall (Fig. 10, green dashdotted), very good agreement is observed between MD and PF. Similar observations we have also made with the VOF method, see Figure 10, blue dotted line, in which we 
(a)

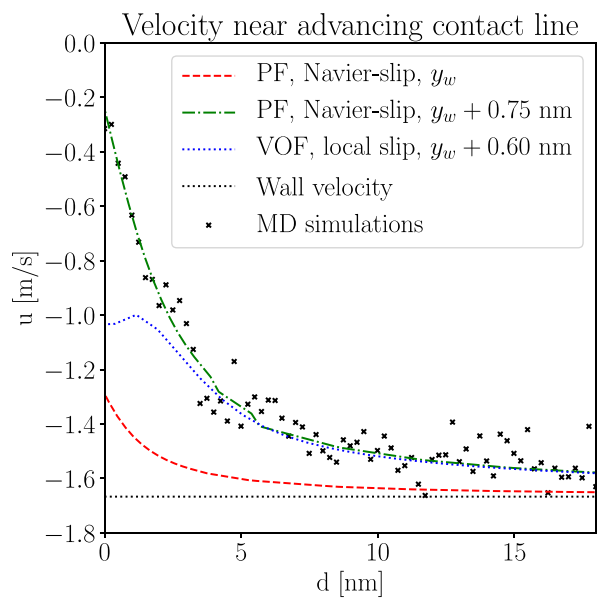

(b)

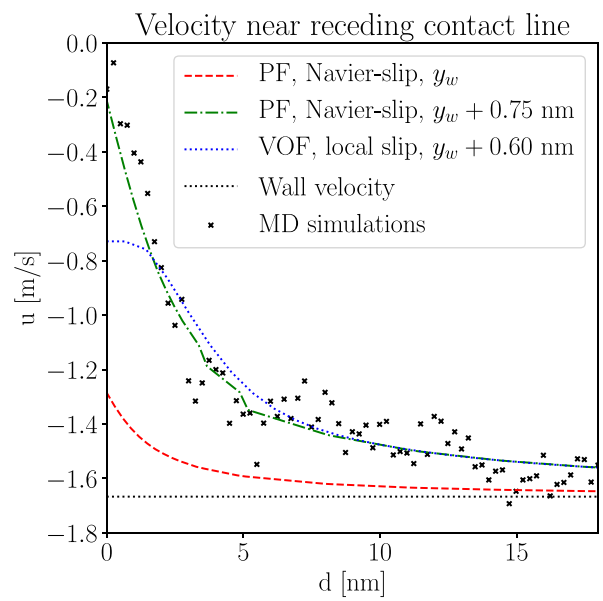

Fig. 10. Velocity near advancing (a) and receding (b) contact lines as a function of $d-$ the distance from the contact line. MD results are compared with PF results (providing best streamlines) at two different vertical sampling locations. and with VOF results at one vertical sampling location.

present VOF results sampled $0.60 \mathrm{~nm}$ away from the wall. This location provides the best agreement with the PF results away from the wall and away from the contact line. Moving the sampling location up or down would yield a similar effect as demonstrated using the PF. Here, an interesting observation is that the VOF flow field seems to be less sharp compared to the PF, which probably stems from the fact that mesh resolution near the interface in $\operatorname{VOF}\left(\Delta s_{V O F}=0.782 \mathrm{~nm}\right.$, Appendix C.1) is much coarser than the resolution of the PF model $\left(\Delta s_{P F}=0.195 \mathrm{~nm}\right.$, Appendix B.2).

These observations raise two important questions about the presented benchmark. First, the Navier-slip condition - based on the slip length measured from the MD $\left(l_{s}=0.17 \mathrm{~nm}\right)$ - does not seem to produce accurate slip velocity even more than $10 \mathrm{~nm}$ away from the contact line. This naturally leads to a question about where the solid wall in the continuum modelling viewpoint should be located, compared to the MD molecular picture (Fig. 2). The wall location is important for application of the Navier-slip condition, as displacement of the wall would cause a direct influence on the needed slip length. Previous studies $[48,49]$ have found that the liquid structure near a solid surface exhibits layering effect, which is an additional hint to a nontrivial correspondence between continuum models and molecular reality. The velocity profile agreement at $0.75 \mathrm{~nm}$ distance from the wall in the continuum simulation seem to suggest that for the best agreement between MD and PF, the wall in the PF simulations should be shifted downwards by $0.75 \mathrm{~nm}$ corresponding to the center of the first bin in Figure 2a. The other option would be to use much larger slip length at the wall (larger by a factor of 4 compared to what is currently measured in the MD). The same discussion applies also to the VOF results with slightly different shift of the wall. The slip and wall location therefore remain open questions.

\subsection{Diffusion of phase-field model}

The Péclet number in theory exists in the MD system, because one can define a selfdiffusion or a heat diffusion coefficient. For the currently used MD water model, the 
self diffusivity is [22]

$$
D_{w}=2.3 \cdot 10^{-9} \mathrm{~m}^{2} / \mathrm{s} .
$$

However, the MD system has a single species and thus has no mass diffusion. By "mass diffusion" we mean that one species diffuses relative to the other, that is if you consider the mass $m_{1}$ of one of the species, or its density $\rho_{1}$, it obeys a diffusion equation.

The PF model in the current work, on the other hand, is incompressible and consequently does not contain any state, energy or heat equation and does not model any self diffusion. The PF model is, however, based on the diffusion of the phasefield variable $C$, see equation (1), and consequently models mass diffusion of liquid. This leads to mismatch between the parameters used in the PF model and diffusion properties determined from the MD system. The Péclet number determined using the self diffusion of the water, relative wall velocity and height of the water drop is

$$
P e=\frac{2 U_{w} L}{D_{w}}=\frac{20 \cdot 50 \cdot 10^{-9}}{6 \cdot 2.3 \cdot 10^{-9}}=72.5
$$

while maximum value we have been able to use in PF simulations and represent the MD results accurately is an order of magnitude smaller, i.e. $P e=6$ (see Tab. 3).

In our opinion, the reason we need to have a moderate $P e$ number in the PF is twofold; (i) the Pe number can not be tow small because we need to capture the phenomena of very small cross-interface mass transport (equivalent to no evaporation), but on the other hand (ii) $P e$ number can not be too large because it also controls the amount of diffusive transport very close to the contact line and controls the finally obtained drop displacement. Nevertheless, due to differences in physical effects MD and PF capture, a perfect agreement in results should not be expected.

\subsection{Density variation of diffuse interface}

Related to the PF diffusion, as discussed in Section 5.3, another interesting question is the shape of density profile exactly at the interface. In order to gain an insight in this question, we have performed a smaller MD simulation (consisting of $10 \mathrm{~nm} \times$ $10 \mathrm{~nm}$ water droplet) of exactly the same water model in static conditions, i.e., between two stationary walls (Appendix A). The obtained density variation at each vertical location has been centred to half of the water density and the mean has been computed between all the vertical locations and all data points in time. The result is shown in Figure 11a with black crosses for 10 ps averaging window. For this time window the shift from vapor to bulk density occurs over $0.7 \mathrm{~nm}$, matching the value of $0.75 \mathrm{~nm}$ reported in Section 2.2 where the used bin sizing was $0.25 \mathrm{~nm}$.

What this distribution captures for the current MD water model is essentially thermal fluctuations of the water-vapour interface, as well as small scale capillary waves. Consequently, this distribution is not invariant with respect to the averaging window. We illustrate this by showing two more density distributions in Figure 11a. When the averaging window is increased in time, the interface has more time to fluctuate over a larger distance and consequently the interface appears more diffuse.

To assess if the diffuse interface in the PF method can be quantitatively matched with the results we obtained from MD, we recall from the PF theory [43] that in the equilibrium the phase function $C$ can be expressed analytically as

$$
C=\tanh \left(\frac{x}{\sqrt{2} \epsilon}\right),
$$


(a)

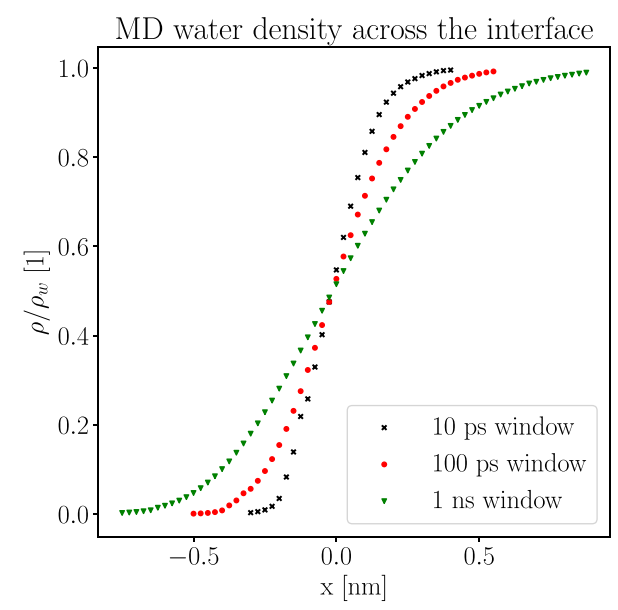

(b)

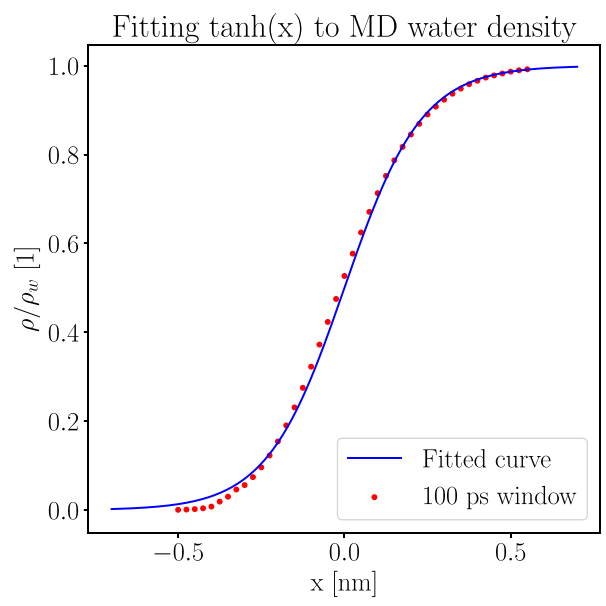

Fig. 11. Density variations across the interface from MD with various averaging windows (a) and PF equilibrium fit to MD data with 100 ps averaging window. The MD data is centred at one half of the water density.

where $x$ is distance from the midpoint of the interface. We use this function in conjunction with the definition of the density (2), set the vapour density to zero $\rho_{g}=0$ and fit the $\epsilon$ parameter to match the MD results with 100 ps averaging window. The obtained fit is shown in Figure 11b. The agreement is very good.

This fit, however, does not have a direct correspondence to the PF diffuse interface, which in theory would describe a region where two liquid species are mixed an have an intermediate density. In the MD water model, however, the density profile in this interpretation is perfectly sharp, i.e., there is no region of water/water-vapour mix having intermediate density. This comparison, however, shows the potential of the PF model to describe the diffusiveness due to thermal fluctuations and/or capillary waves. More detailed investigations are needed to determine the extent of such applicability. For example, it is not clear how large the averaging window should be chosen and if this interpretations holds all the way down to the contact line.

\section{Conclusions}

We have carried out simulations of steady water drop sheared between two moving plates using of MD, PF and VOF methods. The MD simulations allow us to probe the detailed physical picture of the moving contact line and also global measures such as drop displacement amplitude, interface shape and flow field.

By comparing the results from $\mathrm{PF}$ simulations to MD we have observed that a perfect match is not possible. There is however a choice of parameters and boundary conditions which provide a reasonable approximation of MD. We observed a trade-off between accuracy of interface shape and accuracy of the flow field. The best match with respect to the flow field provided the largest error in the interface shape and vice versa. Particularly interesting conclusion from this study is that the exact boundary condition for the flow in the PF simulation does not seem to play a major role. On the other hand, we have observed that for accurate predictions there is an upper limit of the Péclet number one can employ $(\mathrm{Pe}=6.0)$. This is an important insight if one considers using PF model as a substitute for nanoscale simulations, as proposed 
by Kronbichler and Kreiss [50]. This is also in contrast to previous drop spreading $\mathrm{MD}$ and PF comparisons [22], where $\mathrm{Pe}=1400$ was used. This suggests that the PF parameters analysed here are not universal and depend on whether the contact line is advanced through forced wetting or capillary spreading. We have also concluded, that the GNBC, which in the literature is proposed as a solution for the moving contact line problem, is not necessary to model the selected configuration.

Based on the results from PF and MD comparison, we have decided to only investigate a localised Navier-slip boundary condition for the VOF method. With this approach, we managed to capture the drop displacement accurately, while the interface shape had similar local errors as observed in the PF model.

By comparing the bottom water layer velocity from MD with the results of $\mathrm{PF}$ and VOF, we have identified an interesting open question about the accuracy of the slip condition and the wall location. It is possible that from the perspective of the continuum modelling of such small systems, the wall location might play even more important role than the chosen boundary condition. We have discussed the role of diffusion in PF model and the physical phenomena it models, which is different to what happens in MD simulations, therefore a perfect agreement should not be expected. Another possible future direction would be to investigate the width of the localised Navier-slip condition in the VOF model and its influence on the agreement between VOF and MD. Furthermore, comparing the transient behaviour between the $\mathrm{MD}$ and continuum methods would provide additional insight into the robustness of the obtained continuum parameters.

The results of this study will lay rigid foundations for continuum models of moving contact line in realistic water/no-slip substrate two phase systems. We believe that this is the essential first step towards obtaining predictive and robust moving contact line model, suitable for many real world applications.

Open access funding provided by Royal Institute of Technology. U.L. and S.B. acknowledge funding from Swedish Research Council (INTERFACE center and grant nr. VR-2014-5680). S.B. acknowledges funding from Knut and Alice Wallenberg Foundation (Grant Number KAW 2016.0255). Numerical simulations were performed on resources provided by the Swedish National Infrastructure for Computing (SNIC) at PDC and HPC2N. U.L. acknowledges Dr. Minh Do-Quang for sharing his femLego code and assisting with starting up the PF simulations. All authors acknowledge INTERFACE centre for providing collaborative environment and making this project possible. U.L., B.H., G.A. and S.Z. acknowledge the participants of nawet19 workshop for stimulating and interesting discussions.

\section{Author contribution statement}

The idea of this study was conceived by S.B., S.Z., G.A., B.H. and U.L.; the paper was written by U.L., S.Z., P.J. and T.F. with feedback from all authors. MD simulations were carried out by P.J., PF simulations were carried out by U.L. and T.F. carried out VOF simulations. All authors analysed the obtained results.

Open Access This is an open access article distributed under the terms of the Creative Commons Attribution License (http://creativecommons.org/licenses/by/4.0), which permits unrestricted use, distribution, and reproduction in any medium, provided the original work is properly cited.

Publisher's Note The EPJ Publishers remain neutral with regard to jurisdictional claims in published maps and institutional affiliations. 


\section{Appendix A: Details of molecular dynamics simulations}

The system described in Section 2 and Figure 2 consists of two parts: the water slab and the moving walls. The water molecules forming the slab are modeled as SPC/E water [51]. This is a relatively cheap water model which retains important qualities of real water: the three-point structure and a dipole moment. These features gives the ability for the molecule to form hydrogen bonds with other molecules, which leads to a very low slip length $\left(l_{s}=0.17 \mathrm{~nm}\right.$, which is smaller than a molecular diameter) and high surface tension.

The walls are built of rigid $\mathrm{SiO}_{2}$ molecules. Partial charges $q_{\mathrm{Si}}=-2 q_{\mathrm{O}}$ are set to the atoms, making the molecules overall charge neutral but with a quadrupole moment. This electrostatic interaction works with the water molecules to create the aforementioned hydrogen bonding. The charge value $q_{\mathrm{O}}=-0.40 e$ is used to yield the static contact angle $\theta_{0}=97.5^{\circ}$. The molecules are set in an fcc (111) structure with a spacing of $0.45 \mathrm{~nm}$. They are kept in the desired formation by applying harmonic restraints with spring constant $k=10,000 \mathrm{~kJ} \mathrm{~mol}^{-1} \mathrm{~nm}^{-2}$ to the oxygen atoms of each molecule. The molecules are thus free to rotate in the surface normal plane. To move the walls at a desired speed we shift these restraining positions which pulls the molecules along with them while still allowing for natural thermal motion. Remaining details about the wall are described in [22].

Simulations are performed using Gromacs 2019 [52] in double precision. Atomic positions and velocities are updated using the leap-frog integrator with a time step of 2 fs. Non-bonded van der Waals interactions are treated fully up to a cutoff of $0.9 \mathrm{~nm}$. Coulomb interactions are treated using PME electrostatics which interact over infinite range, including all periodic images of the system. Periodic boundary conditions are applied along $x$ and $z$. Along the $y$ axis reflecting walls are placed at each end to contain molecules to the system, although water molecules are kept from reaching these reflecting walls by the physical $\mathrm{SiO}_{2}$ walls. A velocity rescaling thermostat is applied to the $\mathrm{SiO}_{2}$ walls to dissipate excess energy with a time scale of $10 \mathrm{ps}$ and keep the system at simulation temperature $T=300 \mathrm{~K}$. Outside of the initial 100 ps equilibration period, the thermostat is not applied to the water molecules which can only dissipate energy by interacting with the walls through friction. The size of the full simulation domain along $x$ is $150 \mathrm{~nm}$, twice that of the water slab. We have checked that the dissipation of the heat through walls is sufficient to keep the average droplet temperature near $T=300 \mathrm{~K}$. For evaluating the initial equilibration of the system, we have used the water vapour pressure and density from the literature [45] to compute the average number of water molecules in the vapour phase at the equilibrium, which for the current system size yield 15.2 molecules on average. This is roughly the amount we see in vapour phase after equilibration. The reason why the system equilibrates in such a short time is that the evaporation is a surface effect.

Whereas a continuum model can be purely two-dimensional, a molecular system is naturally three-dimensional. A quasi-2D system is created with a system thickness of $4.667 \mathrm{~nm}$ in addition to the domain width and height. With this thickness, a width of $75 \mathrm{~nm}$ and height $50 \mathrm{~nm}$, the water slab for the simulation is constructed with $\sim 580,000$ water molecules.

The small simulation used to determine the water density variation over the interface (Sect. 5.4) is modified as follows. The binning resolution has been increased from the previous $0.25 \mathrm{~nm} \times 0.25 \mathrm{~nm}$ (Fig. $2 \mathrm{~b}$ ) to $0.025 \mathrm{~nm} \times 0.025 \mathrm{~nm}$ to smoothly capture the density variation. The data in each bin is sampled over a time interval of $10 \mathrm{ps}$ and the density variations are captured from this data. The simulation is run over 32 ns to obtain sufficient sampling of the density variation, which is the average of 3200 samples. To obtain the results for larger average windows, we have combined 
10 and 100 of the original samples for 100 ps and 1 ns averaging windows, respectively, in one sample. This construction does not shift the profile of each individual profile to the center position, and therefore produces more spread of the interface. Then final result is obtained from centred average of 320 and 32 samples for 100 ps and $1 \mathrm{~ns}$ averaging windows, respectively.

\section{Appendix B: Details of phase-field simulations}

In this appendix, we describe in more details the non-dimensional governing equations as well as numerical implementation of these equations.

\section{B.1 Dimensionless equations and dimensionless numbers}

In order to render the dimensional governing equations (1), (3) and (4) and the corresponding boundary conditions dimensionless, we choose the relative plate velocity $U=2 U_{w}$, the drop height $L$ and the ration $U / L$ as characteristic scales for velocity, length and time, respectively. Under this assumption, the convection-diffusion equation for phase-field variable $C$ (1) becomes

$$
\frac{\partial C}{\partial t}+\mathbf{u} \cdot \nabla C=\frac{3}{2 \sqrt{2}} \frac{1}{\mathrm{Pe}} \nabla^{2} \phi, \text { with } \phi=\Psi^{\prime}(C)-C_{n}{ }^{2} \nabla^{2} C
$$

where all variables are now dimensionless. This procedure gives rise to Péclet number and Cahn number,

$$
\mathrm{Pe}=\frac{U \epsilon L}{M \sigma} \text { and } C_{n}=\frac{\epsilon}{L}
$$

The Péclet number is quite important as it provides a measure of relative importance between convective and diffusive transport of the $\mathrm{PF}$ concentration function $C$. This number we use in the main text to report the needed PF mobility values for the agreement between PF and MD, see Tables 1, 2 and 3.

The incompressible Navier-Stokes equations (3)-(4) in non-dimensional form becomes

$$
\operatorname{Re} \rho(C)\left[\frac{\partial \mathbf{u}}{\partial t}+(\mathbf{u} \cdot \nabla) \mathbf{u}\right]=-\nabla P+\nabla \cdot\left[\mu(C)\left\{\nabla \mathbf{u}+(\nabla \mathbf{u})^{T}\right\}\right]-\frac{3}{2 \sqrt{2}} \frac{C \nabla \phi}{C_{n} \mathrm{Ca}}
$$

where again all variables now are non-dimensional. During this process, two new dimensionless numbers are introduced, namely Reynolds and capillary numbers, as

$$
\operatorname{Re}=\frac{\rho_{w} U L}{\mu_{w}} \text { and } \mathrm{Ca}=\frac{\mu_{w} U}{\sigma} .
$$

The density and viscosity is normalised with respect to the parameters of water, and therefore in the dimensionless setting becomes

$$
\rho(C)=\frac{1}{2}\left[(C+1)-\frac{\rho_{g}}{\rho_{w}}(C-1)\right], \quad \mu(C)=\frac{1}{2}\left[(C+1)-\frac{\mu_{g}}{\mu_{w}}(C-1)\right],
$$

where $\rho_{g}$ and $\mu_{g}$ are the density and the viscosity of the vapour part, as shown in Figure 1 . 
Finally, the dimensionless wetting condition becomes

$$
-\mu_{f} \frac{2 \sqrt{2}}{3} \operatorname{Ca} C_{n}\left(\frac{\partial C}{\partial t}+\mathbf{u} \cdot \nabla C\right)=C_{n} \nabla C \cdot \hat{n}-\frac{2 \sqrt{2}}{3} \cos \left(\theta_{0}\right) g^{\prime}(C)
$$

Note that the numerical pre-factors $2 \sqrt{2} / 3$ appearing in certain places of the dimensional governing equation stems from relationship between the dimensional phase-field constants $\alpha$ and $\beta$ and the surface tension, i.e., $\sigma=2 \sqrt{2 \alpha \beta} / 3$.

\section{B.2 Numerical implementation}

The dimensionless governing equations introduced in the Appendix B.1 are linearised, cast into the weak form and solved using a symbolic finite-element toolbox femLego [53], which allows easy specification of finite-element weak form to solve. The solver is based on finite-element package deal.II and includes adaptive mesh refinement to resolve the sharp transition of phase-field variables over a thin interface [54]. Linear elements were used for the phase-field variables, while the fluid flow was resolved using Taylor-Hood elements (quadratic for velocity and linear for pressure).

Mesh resolution was selected after a refinement study based on PF simulation with a no-slip condition. The final resolution selected and used for all other simulations is $\Delta s_{1}=3.125 \mathrm{~nm}$ far from the interface, and down to $\Delta s_{2}=0.195 \mathrm{~nm}$ within the interface region. Constant time step was used through the simulation as $\Delta t=0.002$ dimensionless time units.

\section{Appendix C: Volume-of-fluids model}

In this Appendix, we describe in more details the numerical implementation of the solver, the height functions employed for the VOF model, as well as the implementation of velocity boundary condition.

\section{C.1 Numerical implementation}

In our study of the sheared droplet system, we used the free software Basilisk, successor of Gerris, developed at the Institut Jean le Rond d'Alembert (Sorbonne Université) [47,55-57]. The incompressible Navier-Stokes equations are discretised using the finite volume method and are solved using second order Bell-Colella-Glaz projection scheme [58]. The solver is coupled with VOF method for interface tracking. For obtaining the solution, we have used a uniform mesh spacing of $\Delta s=0.782 \mathrm{~nm}$.

\section{C.2 Height functions}

The height-function methodology is a VOF-based technique for calculating interface normals and curvatures. About each interface cell, fluid "heights" are calculated by summing fluid volume in the direction most normal to the interface. In $2 \mathrm{D}$, a $7 \times 3$ stencil around an interface cell is constructed (Fig. C.1) and the heights are evaluated 


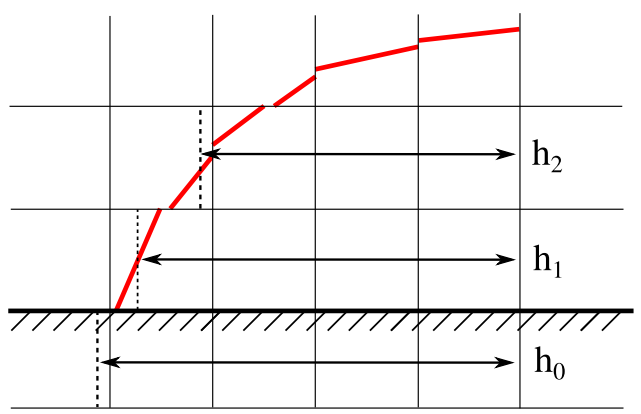

Fig. C.1. Construction of the 2D height-functions.

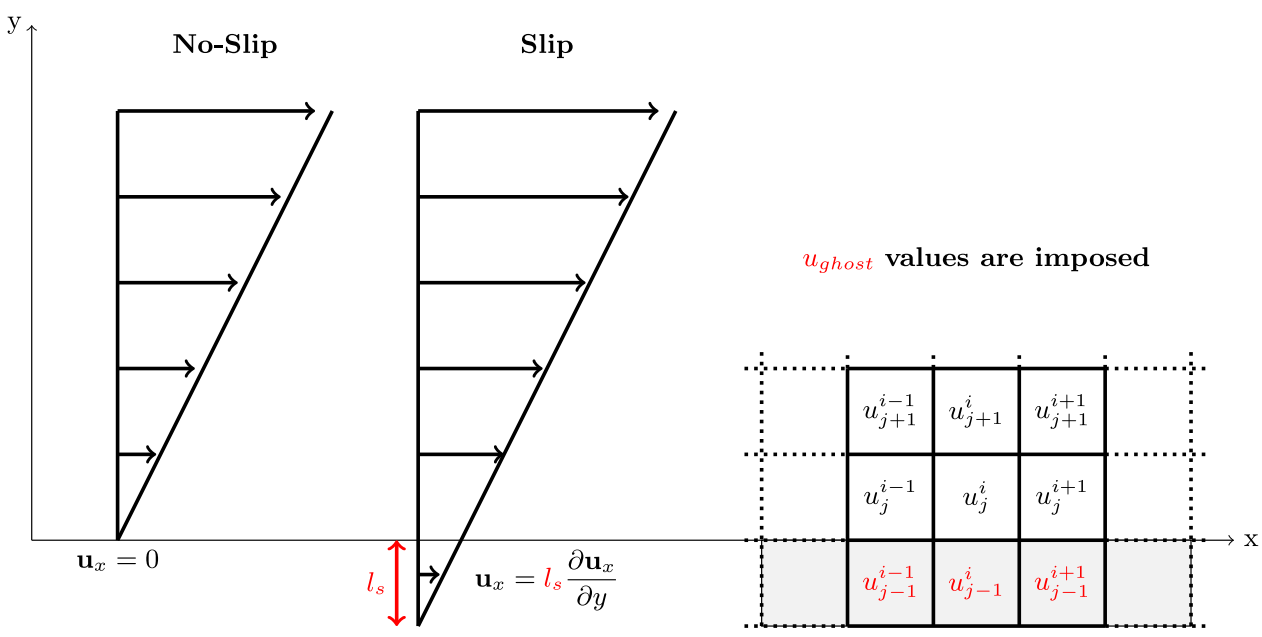

Fig. C.2. Left side : velocities proles at the solid interface for the no-slip and slip boundary conditions. Right side: $3 \times 3$ stencils with the ghost boundary layer used to impose the boundary condition.

by summing volume fractions horizontally, i.e.

$$
h_{i}=\sum_{k=j-3}^{k=j+3} C_{i, k} \Delta
$$

where $\Delta$ is the grid spacing. The heights are then used to compute the the interface normal $\hat{n}$ and the curvature $\kappa$ as

$$
\hat{n}=\left(\partial_{x} h,-1\right) \text { and } \kappa=\frac{\partial_{x x}^{2} h}{\left(1+\left(\partial_{x} h\right)^{2}\right)^{3 / 2}},
$$

respectively. Here, $\partial_{x} h$ and $\partial_{x x}^{2} h$ are discretised using second-order central differences.

\section{C.3 Implementation of velocity boundary condition}

In order to impose the velocity boundary condition at the wall in the selected numerical implementation, one has to work with discretisation stencils near the solid 
boundary. These stencils will extend beyond beyond the wall and make use of so called ghost points (Fig C.2). The stencil values outside the domain (ghost values) need to be initialised. These values are set in order to provide the discrete equivalent of the NBC as

$$
\begin{aligned}
& \frac{\mathbf{u}_{x}[\text { ghost }]+\mathbf{u}_{x}[]}{2}+l_{s} \frac{\mathbf{u}_{x}[\text { ghost }]-\mathbf{u}_{x}[]}{\boldsymbol{\Delta}}=U_{w} \\
& \Longleftrightarrow \mathbf{u}_{x}[\text { ghost }]=\frac{2 \boldsymbol{\Delta}}{2 l_{s}+\boldsymbol{\Delta}} U_{w}+\frac{2 l_{s}-\boldsymbol{\Delta}}{2 l_{s}+\boldsymbol{\Delta}} \mathbf{u}_{t}[]
\end{aligned}
$$

where $\mathbf{u}_{x}[$ ghost $]$ is the tangential velocity at the ghost cell, $\mathbf{u}_{x}[]$ is the tangential velocity of the cell inside the domain and $\boldsymbol{\Delta}$ is the grid spacing. This implementation is used to impose the locally specified Navier-slip condition, presented in equation (11).

\section{References}

1. C. Huh, L.E. Scriven, J. Colloid Interf. Sci. 35, 85 (1971)

2. D. Bonn, J. Eggers, J. Indekeu, J. Meunier, E. Rolley, Rev. Mod. Phys. 81, 739 (2009)

3. J.H. Snoeijer, B. Andreotti, Annu. Rev. Fluid Mech. 45, 269 (2013)

4. Y. Sui, H. Ding, P.D.M. Spelt, Annu. Rev. Fluid Mech. 46, 97 (2014)

5. C.-L. Navier, Mémoires de l'Académie Royale des Sciences de l'Institut de France 6, 389 $(1823)$

6. O. Voinov, Fluid Dyn. 11, 714 (1976)

7. R.G. Cox, J. Fluid Mech. 168, 169 (1986)

8. Y.D. Shikhmurzaev, Fluid Dyn. Res. 13, 45 (1994)

9. Y.D. Shikhmurzaev, J. Fluid Mech. 334, 211 (1997)

10. S. Kalliadasis, C. Bielarz, G.M. Homsy, Phys. Fluids 12, 1889 (2000)

11. J. Eggers, Phys. Rev. Lett. 93, 094502 (2004)

12. J.C. Flitton, J.R. King. J. Eng. Math. 50, 241 (2004)

13. J.H. Snoeijer, Phys. Fluids 18, $021701(2006)$

14. L.M. Pismen, J. Eggers, Phys. Rev. E 78, 056304 (2008)

15. J.H. Snoeijer, J. Eggers, Phys. Rev. E 82, 056314 (2010)

16. T.S. Chan, J.H. Snoeijer, J. Eggers, Phys. Fluids 24, 072104 (2012)

17. A. Nold, L. González MacDowell, D.N. Sibley, B.D. Goddard, S. Kalliadasis, Mol. Phys. 116, 2239 (2018)

18. T.D. Blake, J.-C. Fernandez-Toledano, G. Doyen, J. De Coninck, Phys. Fluids 27, 112101 (2015)

19. Y. Deng, L. Chen, Q. Liu, J. Yu, H. Wang, J. Phys. Chem. Lett. 7, 1763 (2016)

20. M. Fricke, M. Köhne, D. Bothe, Proc. Appl. Math. Mech. 18, e201800451 (2018)

21. M. Fricke, M. Köhne, D. Bothe, Physica D 394, 26 (2019)

22. P. Johansson, A. Carlson, B. Hess, J. Fluid Mech. 781, 695 (2015)

23. P. Johansson, B. Hess, Phys. Rev. Fluids 3, 074201 (2018)

24. N.G. Hadjiconstantinou, J. Comput. Phys. 154, 245 (1999)

25. J. Zhang, M.K. Borg, J.M. Reese, Int. J. Heat Mass Transf. 115, 886 (2017)

26. E.R. Smith, P.E. Theodorakis, R.V. Craster, O.K. Matar, Langmuir 34, 12501 (2018)

27. M.K. Borg, D.A. Lockerby, K. Ritos, J.M. Reese, J. Membrane Sci. 567, 115 (2018)

28. L.M. Pismen, Eur. Phys. J. Special Topics 197, 63 (2011)

29. T.D. Blake, in Dynamic contact angles and wetting kinetics, Vol. 49. Surfactant Science Series, chap. 5 (Marcel Dekker, Inc., 1993), pp. 251-309

30. T. Qian, X.-P. Wang, P. Sheng, Phys. Rev. E 68, 016306 (2003)

31. T. Qian, X.-P. Wang, P. Sheng, J. Fluid Mech. 564, 333 (2006)

32. A. Carlson, M. Do-Quang, G. Amberg, J. Fluid Mech. 682, 213 (2011) 
33. D. Jacqmin, J. Fluid Mech. 402, 57 (2000)

34. T. Laurila, A. Carlson, M. Do-Quang, T. Ala-Nissila, G. Amberg, Phys. Rev. E 85, $026320(2012)$

35. J.J. Eggleston, G.B. McFadden, P.W. Voorhees, Physica D 150, 91 (2001)

36. A. Carlson, M. Do-Quang, G. Amberg, Phys. Fluids 21, 121701 (2009)

37. W. Villanueva, G. Amberg, Int. J. Multiphas. Flow 32, 1072 (2006)

38. D. Jacqmin, J. Fluid Mech. 517, 209 (2004)

39. M. Sbragaglia, K. Sugiyama, L. Biferale, J. Fluid Mech. 614, 471 (2008)

40. W. Ren, W. E, Phys. Fluids 19, 022101 (2007)

41. P.L. Barclay, J.R. Lukes, Phys. Fluids 31, 092107 (2019)

42. H.S.H. Mohand, H. Hoang, G. Galliero, D. Legendre, J. Comput. Phys. 393, 29 (2019)

43. A. Carlson, Capillarity and dynamic wetting, PhD thesis, KTH Royal Institute of Technology, 2012

44. D. Jacqmin, J. Comput. Phys. 155, 96 (1999)

45. Engineering ToolBox, Water Vapor and Saturation Pressure in Humid Air, 2004. [online] Available at: https://www.engineeringtoolbox.com/water-vapor-saturationpressure-air-d_689.html [Accessed 5th of February, 2020]

46. Engineering ToolBox, Gases - Dynamic Viscosity, 2014. [online] Available at: https://www.engineeringtoolbox.com/gases-absolute-dynamic-viscosity-d_1888.html [Accessed 5th of February, 2020]

47. S. Afkhami, J. Buongiorno, A. Guion, S. Popinet, R. Scardovelli, S. Zaleski, J. Comput. Phys. 374, 1061 (2017)

48. I. Bitsanis, T.K. Vanderlick, M. Tirrell, H.T. Davis, J. Chem. Phys. 89, 3152 (1988)

49. H. Hoang, G. Galliero, Phys. Rev. E 86, 021202 (2012)

50. M. Kronbichler, G. Kreiss, J. Comput. Multiphas. Flows 9, 114 (2017)

51. H.J.C. Berendsen, J.R. Grigera, T.P. Straatsma, J. Phys. Chem. 91, 6269 (1987)

52. M.J. Abraham, T. Murtola, R. Schulz, S. Páll, J.C. Smith, B. Hess, E. Lindahl, SoftwareX 1-2, 19 (2015)

53. G. Amberg, R. Tönhardt, C. Winkler, Math. Comput. Simulat. 49, 257 (1999)

54. M. Do-Quang, W. Villanueva, I. Singer-Loginova, G. Amberg, Bulletin of the Polish Academy of Sciences: Technical Sciences 55, 229 (2007)

55. S. Afkhami, M. Bussmann, Int. J. Numer. Meth. Fl. 57, 453 (2008)

56. S. Popinet, Annu. Rev. Fluid Mech. 50, 49 (2018)

57. S. Afkhami, M. Bussmann, Int. J. Numer. Meth. Fl. 61, 827 (2009)

58. J.B. Bell, P. Colella, H.M. Glaz, J. Comput. Phys. 85, 257 (1989) 